\title{
Malaria: Autophagy as a Potential Therapeutic Target
}

\author{
Prati Pal Singh and Purbali Chakraborty \\ Centre of Infectious Diseases, Department of Pharmacology and Toxicology, National Institute of Pharmaceutical Education and \\ Research, India
}

\begin{abstract}
Malaria, where the causative agent Plasmodium is rapidly gaining resistance to practically all the antimalarial drugs in clinical use, requires the identification of new drug targets to enable the discovery of novel, more effective and safer drugs to treat the disease. Advancements in molecular techniques have provided the proof of existence of autophagy in Plasmodium; however, its role(s) in malaria is only becoming to be understood. Nevertheless, some of the recently explored dimensions of autophagy in Plasmodium have indicated its fairly larger role in parasite survival and growth. But then, autophagy is also essential for host cell survival and defence. There is thus need to explore chemotherapeutic strategies to specifically target autophagy in both the parasite and host. This review focuses on autophagy pathways in Plasmodium and in host with a view to identify autophagy-related new drug targets for the discovery of novel antimalarial drugs.
\end{abstract}

Key words: Apicoplast, autophagy, malaria, Plasmodium, therapeutic target.

\section{Introduction}

Malaria is one of the world's major public health burdens due to its huge toll of morbidity and mortality. According to the latest (released September 2015) WHO estimates, in 2015, there were around 3.2 billion people at the risk of malaria, and there were 214 million clinical cases and 438, 000 deaths [1]. Human malaria is caused by six different species of the protozoan parasite Plasmodium, which are Plasmodium falciparum, P. vivax, P. malariae, $P$. ovale, P. knowlesi [2] and P. brasilianum [3]; all of them transmitted by the bite of female Anopheles mosquitoes.

In most regions of the world, chloroquine is viewed to be ineffective against $P$. falciparum, and there are reports of clinical treatment failures for most antimalarial drugs currently in clinical use [4]. Presently, artemisinin-based combination therapy (ACT) is considered the best treatment option for the uncomplicated malaria caused by $P$. falciparum [5].

Corresponding author: Prof. Prati Pal Singh, Center of Infectious Diseases, Department of Pharmacology and Toxicology, National Institute of Pharmaceutical Education and Research, Sector 67, Phase-X, S. A. S. Nagar-160 062, Punjab, India.
But, there is now growing evidence for the declining efficacy of some of the ACTs, such as artesunate-mefloqine at the Cambodia-Thailand border [6]. The therapeutic treatment and control of malaria has become more challenging due to growing parasite resistance to current antimalarial drugs, most crucial being the emergence of $P$. falciparum resistance to artemisinins, which is an urgent public health concern and imperils the ongoing global efforts to reduce the burden of malaria [7]. Therefore, there is an urgent need to discover and develop newer and effective antimalarial drugs that act on novel targets within the parasite, and thus, in all probability, ensure a hitherto unknown mechanism of action.

To maintain cellular homeostasis, the eukaryotic cells have developed specialised mechanisms such as lysis of intracellular proteins and organelles, which regulate cellular functions like enzymatic activity, removal of toxic or misfolded proteins, and the production of free amino acids to ensure cell survival under stressful conditions. The eukaryotic cells are known to perform these functions by the process of autophagy which is believed to have originated at a later point during evolution [8]. Over millions of years of co-evolution and co-existence, malaria parasites and 
human beings have developed several adaptations; one such adaptation being the complex processes known as autophagy. Homologues of many of the genes which regulate autophagy in humans have been reported in $P$. falciparum [9]. Autophagy is a mechanism for the degradation of cellular components in the cytoplasm, and it serves as a cell survival mechanism under stressful conditions [10]. The effects of autophagy on the pathogenesis and progression of malaria are apparently not yet known clearly and in entirety. The identification of new enzyme targets in the autophagy pathway in Plasmodium, and to exploit them to discover novel antimalarial drugs continues to be a key focus and a major challenge.

\section{Autophagy: A Cell Survival Mechanism}

The term autophagy, derived from the Greek words 'auto' (self) and 'phagy' (eating) was first mentioned by Christian de Duve in 1960s [11]. Autophagy is a key cellular homeostatic process which regulates cellular clean-up of cytoplasmic portions and degrade their constituents. The process of autophagy begins with the formation of an isolation membrane, which then encloses certain portions of the cytoplasm, leading to the formation of a double membrane organelle, termed as the autophagosome. The enclosed cytoplasmic material in the autophagosome gets degraded when autophagosome fuses with the late endosomes or lysosomes [12], or vacuoles in yeast [13]. Autophagy is mostly a cytoprotective, catabolic process which also functions in cell death, but its precise role(s) in cells undergoing death is not clear [14]. Several types of autophagic processes have been suggested till date, and these are macroautophagy [15], microautophagy, chaperone-mediated autophagy, and the other related processes like LAP (LC3-associated phagocytosis), autophagic degradation of cellular organelles such as mitochondria (mitophagy), peroxisomes (pexophagy), endoplasmic reticulum (reticulophagy), ribosomes (ribophagy), autophagic degradation of ubiquitinated protein aggregates (aggrephagy) and pathogens (xenophagy), autophagy for antigen presentation and selectively directing inflammosome components and pro-inflammatory cytokines (inflammophagy and cytokinophagy) [16]. The term autophagy typically refers to macroautophagy, if not delimited [12].

\section{Autophagy in Plasmodium}

De Brito et al. (1969), for the first time, reported autophagy in $P$. falciparum- and P. vivax-infected human liver tissues. They observed the presence of several vacuoles of various sizes, defined by single or double membranes, and identified those bodies as malaria pigment [17]. Chloroquine (a lysosomotropic agent which increases lysosomal $\mathrm{pH}$ and inhibits autophagy) has been reported to produce prominent structural changes in lysosomes, and within 30 minutes of its administration many autophagy vacuoles have been observed [18]. This was probably due to the inhibition of autophagy by chloroquine, which resulted in the accumulation of vacuoles of autophagy. Totino et al. (2007) have demonstrated that under choroquine treatment, $P$. falciparum blood-stage parasites die by a process similar to autophagy, as confirmed by the observation of cytoplasmic vacuoles [19]. Nevertheless, only the visualisation of autophagy vacuoles observed through electron microscopy does not confirm the occurrence of autophagy [20]. However, information is now available regarding the functioning of autophagy in P. falciparum despite the absence of a typical lysosome for fusion with autophagosomes [21].

All the three forms of cell death: apoptosis (type I), autophagy (type II) and necrosis (type III) have been suggested to occur in P. falciparum [22]. However, if only we can identify and understand the molecular mechanisms behind these cell death pathways, it may be possible to trigger them to eliminate the parasite. Plasmodium is known to have conserved certain aspects of autophagy pathway, and homologues of some of the proteins essential for macroautophagy have been found in Plasmodium [23]. But the actual purpose of autophagy in Plasmodium, though not yet 
properly and fully known, appears to be quite different from canonical autophagy. Some of the roles of autophagy proteins (Atg) in Plasmodium have been suggested, like vesicular trafficking (including protein secretion, organelle expulsion and trafficking to food vacuole), programmed cell death and apicoplast maintenance [24], though the roles of several plasmodial Atg continue to remain unknown. In case of protein secretion, it is known that Plasmodium, after entry into host cell, carries out extensive remodelling of the host cell by exporting its own proteins into host cell through parasite plasma membrane and the parasitophorous vacuole. The process of exophagy (non-conventional protein secretion pathway), which involves movement of autophagosomal proteins to plasma membrane might occur in this case, where the role(s) of some of the Atg has been demonstrated [25]. In case of organelle expulsion, it is known that during the morphological transformation of Plasmodium in the early liver stages, certain organelles used for liver cell invasion (rhoptries, micronemes and others) get expelled from parasite. The presence of double-membraned structures, morphologically similar to autophagosomes containing micronemes, indicates that exophagy might be important for parasite differentiation in liver stages [22]. Atg8 is an autophagy marker protein which remains attached with the pre-autophagosome and autophagosome during lysosomal fusion. Vesicular trafficking of haemoglobin and other nutrients from host erythrocytes to plasmodial food vacuole is also known as an important process for parasite survival, where the involvement of P. falciparum Atg8 (PfAtg8) has been suggested [25]. Prolonged treatment with chloroquine has been reported to result in the accumulation of such haemoglobin-containing vesicles, which do not fuse with digestive vacuoles, and result in the prevention of haemoglobin digestion and amino acid starvation of malaria parasite [21]. Under the treatment of autophagy inhibitors like chloroquine or wortmannin, no change was observed in the PfAtg8 localization [26]. However, chloroquine treatment at cytocidal concentration has been shown to provoke PfAtg8 relocalization to RBC cytoplasm in chloroquine-sensitive strains, which gets significantly altered in chloroquine-resistant strains [27]. These observations suggest that PfAtg8 may be important for vesicular trafficking of haemoglobin. Gaviria et al. (2013) have reported that a process similar to autophagy is associated with the emergence of resistance against cytocidal doses of chloroquine against intra-erythrocytic $P$. falciparum trophozoites [28]. In several studies involving cancer cell-lines, the antimalarial artemisinin and its metabolite dihydroartemisinin have been reported to induce autophagy. The results of these studies suggest excessive intracellular reactive-oxygen species production as one of the probable mechanism of autophagy induction, which, in turn, further enhances the apoptotic response in such cells [29, 30]. The generation of free-radicals leading to the reduction of $\mathrm{RBC}$ antioxidants and glutathione is considered to be one of the antimalarial mechanisms of action of dihydroartemisinin [31].

Mizushima and Sahani (2014) have shown that PfAtg8 is usually located at the apicoplast membrane of Plasmodium throughout liver- and blood-stages, but there is no evidence of autophagic membranes surrounding apicoplast [32]. This contention suggests that Atg8 may not be involved in the autophagic degradation of apicoplast. Hain and Bosch (2013) have reported that rapamycin (an autophagy inducer) treatment $(500 \mathrm{nM})$ of malaria parasite ensues in the reduction of its size and change in the morphology of apicoplast. Surprisingly, rapamycin treatment did not affect $P$. berghei Atg8 (PbAtg8) localisation, which might be possible because of the absence of the target of rapamycin orthologue in $P$. berghei, and might be mediated through its effects on host autophagy or through other targets [25]. In another study, rapamycin has been shown to inhibit the growth of $P$. falciparum, in vitro, at concentrations of $7.2 \mu \mathrm{mol} / \mathrm{L}\left(\mathrm{IC}_{50}\right)$ and 12.5 $\mu \mathrm{mol} / \mathrm{L}\left(\mathrm{IC}_{90}\right)[33]$. 
Tomlins et al. (2013) have shown that in the erythrocytic stage, under amino acid deprivation, PfAtg8-containing structures (likely autophagosomes) fuse with $P$. falciparum Rab7 (PfRab7)-containing vesicles (likely late endosomes) to generate acidified vesicles. These vesicles, in turn, fuse with the digestive vacuoles of parasite (the final lytic compartment) which resembles lysosomal network of higher eukaryotes. Rab7 is a small GTP-binding protein important for late endosomal transport, lysosomal biogenesis and, hence, progression of autophagy. This view-point suggests that under starvation, $P$. falciparum may use the food vacuole for an autophagy-like process, and undergoes transcriptionally silent hibernation-like state, which might be responsible for the remodelling of subcellular organelles to remove dispensable cellular material during intra-erythrocytic growth [21]. In a similar study, Cervantes et al. (2014) have shown that under mild nutrient starvation, autophagy cannot be induced in P. falciparum. This observation supported the absence of an increase in the amount of PfAtg8-containing autophagosomes, which indicates that unlike mammalian autophagy, P. falciparum lacks the genes required for nutrient-dependent autophagy induction [24].

PfAtg8 has been implicated in both autophagy and apicoplast maintenance [21]; however, the actual mechanism of PfAtg8 in apicoplast maintenance remains unknown. To understand the underlying mechanism, there is need to determine the effect of various inhibitors of apicoplast biosynthetic pathways on the growth of $P$. falciparum, sufficient or deficient in PfAtg8. One such study has reported that PfAtg7 ( $P$. falciparum Atg7, an autophagy protein essential for the normal growth of parasite)-attenuated parasites are relatively more sensitive to fosmidomycin (an inhibitor of apicoplast isoprenoid biosynthetic pathway) [34], which indicates that PfAtg7 is essential for properly functioning apicoplast, and PfAtg7 inhibitors can be developed as a novel class of potential antimalarial drugs.

Before the beginning of the blood-stage of malaria infection, sporozoites continue to develop in liver, wherein parasites undergo sporozoite-to-trophozoite differentiation by the elimination of certain organelles like micronemes and rhoptries, which get enclosed within double-membraned structures similar to autophagosomes. These observations suggest the occurrence of autophagy during parasite morphological remodelling [35], and have been supported by the observations made following parasite treatment with 3-methyladenine, an autophagy inhibitor that targets Vps34 (class III phosphoinositide 3-kinase, essential for membrane trafficking process during autophagy), which significantly retarded the sporozoite differentiation process [36]. Similarly, $P$. berghei $\operatorname{Atg} 8(\mathrm{PbAtg} 8)$ is also known to be involved in organelle degradation (microneme disposal) via autophagy and in vesicular trafficking between organelles during intrahepatic development. However, PbAtg8 may not be involved in autophagosome formation in the liver stage of the P. berghei, because it remains localised exclusively to the apicoplast membrane, and remains associated with the functioning of mitochondrion in parasite [22]. Therefore, PbAtg8 cannot be considered as a typical autophagy marker during intrahepatic stages of $P$. berghei. Hence, there is a need to understand the molecular mechanisms of autophagy in plasmodial liver-stages in a more comprehensive manner to enable to target its components, specifically, which result in parasite death, including even the death of dormant hypnozoites.

\section{Autophagy in Host}

Autophagy in hosts evolved along with the disease causing organisms in a contemporaneous manner, as a selective pressure and cellular mechanism of protection from such microbes. It is, therefore, considered an antimicrobial defence mechanism that can degrade intracellular pathogens. However, the physiological 
and the pathophysiological roles of autophagy in mammalian cells are vast, like starvation adaptation, transportation (sequestration and packing), intracellular protein and organelle clearance, development, antiaging, elimination of microorganisms, cell death, tumour suppression, in innate immunity, and in adaptive immunity [12].

Autophagy plays vital roles in the survival, maintenance, functioning and death of the cells of immune system. Another major role of autophagy recognised till date is in antigen presentation to MHC (major histocompatibility complex) class II molecules, and their further recognition by $\mathrm{CD}^{+} \mathrm{T}$ cells [37]. For antigen presentation to take place, endocytosed materials need to be transported to lysosomes, and their fragments then presented to T cells. Phagocytosis, an endocytosis pathway, is known to be regulated by Atg, particularly during LC3 (microtubule-associated protein $1 \mathrm{~A} / 1 \mathrm{~B}$ - light chain 3 , an autophagosomal membrane protein)-associated phagocytosis [38]. The autophagy components (particularly Atg5) regulate the process of phagocytosis in such a way that it appears to enhance the MHC class II antigen presentation [39]. Atg also regulates the secretion of some of the immune mediators elaborated by macrophages such as IL $1 \alpha$ and IL1 $\beta$, which indicates that autophagy in macrophages is a potent regulator of inflammation [40].

Autophagy is also involved in the elimination of macromolecules and cellular organelles including intracellular pathogens. It has been already reported that autophagy is involved in the restriction of the replication, growth and survival of intracellular pathogens (viral, bacterial, protozoal and fungal) [41-44]. This type of autophagy has been selectively termed as xenophagy. In case of Mycobacterium tuberculosis infection, when autophagy was induced in infected macrophages, there was restoration of phagosome maturation and mycobacterial killing [45]. The LC3 protein, a mammalian orthologue of Atg8 subfamily and an autophagosome membrane protein, can engage in xenophagy against Salmonella in the absence of upstream Atg proteins [46].

During infections, many aspects of autophagy pathway have been suggested like the regulatory pathways [PRRs (pathogen recognition receptors), PAMPs (pathogen associated molecular patterns), and downstream signalling pathways], xenophagy of microbes, and the events in autophagosome formation during infection [47]. As has been already discussed, innate immune mechanisms of autophagy restrict infection by removing the microbes from cytosol through lysosomal degradation as well as by other aspects of innate and adaptive immunity. Innate immunity signalling pathways also regulate autophagy induction, and autophagosomes may help to deliver ligands for innate and adaptive immune activation. One of the defence mechanisms against invading pathogens is the recognition of PAMPs by PRRs, including TLRs (Toll-like receptors), and these essential innate immune receptors have also been reported to regulate autophagy [48]. Apart from the recognition of PAMPs by PRRs, there is a complex interplay between other immune signals and autophagy regulation. Autophagy itself is known to be regulated by different immune signalling pathways [49].

In case of $P$. berghei sporozoite-infected hepatocytes, lipidation and incorporation of LC3 into PVM (parasitophorous vacuolar membrane) have been observed, which incite an autophagy-like pathway to eliminate parasites [50]. It has also been suggested that genetically attenuated $P$. berghei sporozoites, which are unable to produce a mature PVM within hepatocytes, might get readily detected by the host cell, which in turn, may trigger xenophagy [51]. So, the attenuated sporozoites may survive as long as the defence mechanisms of host cell allow, or as long as the sporozoites themselves can nullify the host cell defence mechanism. Further, in a mice model of ECM (experimental cerebral malaria), rapamycin treatment has been reported to protect against ECM by reducing the migration of $\mathrm{CD} 4+$ and $\mathrm{CD} 8+\mathrm{T}$ cell to the brain, which protects the blood-brain barrier and activates the 


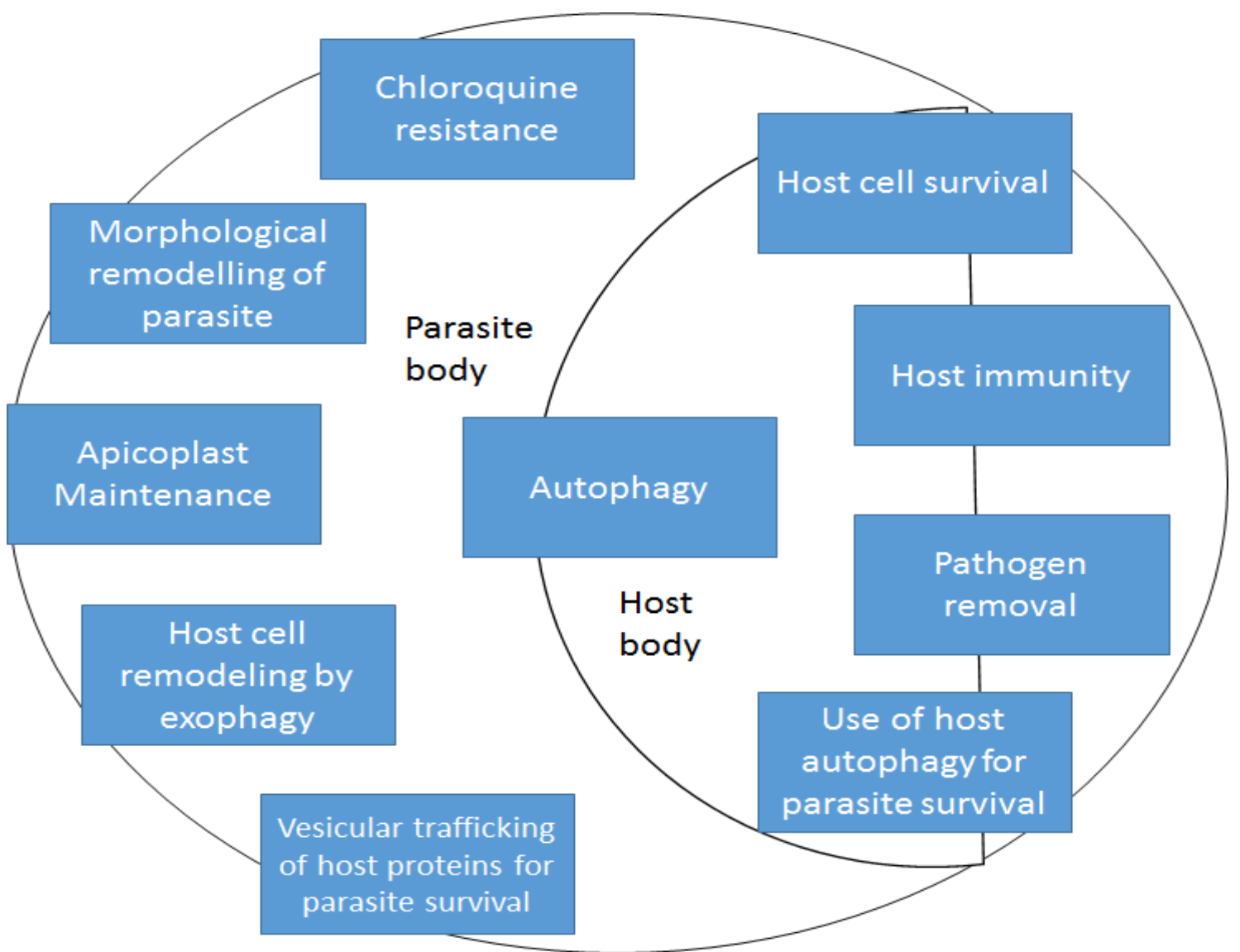

Fig. 1 Autophagy in the pathogenesis of malaria.

macrophages for the augmented clearance of infected-RBCs [52]. Thus, during malaria, autophagic defence may protect host cells from infection.

Because autophagy has been suggested to be a significant host defence mechanism, microbes also undergo strong selective pressure under its influence, and develop mechanisms to evade host autophagic defence. Such instances have been observed in case of HIV and M. tuberculosis infections [48]. During schizogony, many of the Plasmodium parasites are able to evade the host cell autophagic defence by removing LC3 from PVM (LC3 targeting of PVM is a common feature of autophagy-related processes which occur during LC3-associated phagocytosis), and hence avoid lysosomal attack, while obtaining additional nutrients from host cell. Thus, it appears that a balance exists between the parasite and the host cell to regulate the extent of infection [50]. P. yoelii sporozoites have also been shown to disturb hepatocyte autophagic survival pathways [53]. Thus, taken together, all these observations suggest that a proper understanding of host autophagy and its interaction with Plasmodium (Fig.1), may help us to find newer and novel targets to discover and develop new therapies for malaria.

\section{Conclusions and Future Prospects}

The emergence of malaria parasite strains resistant to drugs in clinical use draws out the necessity to discover and develop new drugs, especially those which act on novel targets. The process of autophagy, which is essential for parasite survival, virulence, and growth, and for host as well, is an expedient target of interest. The plasmodial autophagy, which appears to be different from that of mammalian autophagy, is considered responsible for the vesicular trafficking of parasite proteins into host cell, which results in the remodelling of host cell and parasite organelle expulsion. These two seemingly different but related 
biological events, in turn, ensue in morphological transformation of malaria parasite in hepatic stages and thus ensure parasite differentiation. Therefore, the inhibitors of both these processes can be developed as potential novel antimalarial compounds, which may plausibly exert their antimalarial action by the restriction of parasite differentiation and growth. The chloroquine inhibition of autophagic vesicular trafficking of haemoglobin from host RBC to parasite food vacuole, and the consequent energy starvation of the parasite indicates that similar compounds which target haemoglobin trafficking, particularly the inhibitors of PfAtg8, can be designed, identified and developed as potential novel antimalarials. The maintenance of parasite apicoplast by PfAtg8 suggests that compounds which target autophagy-related apicoplast biosynthetic pathways can also be expected to have potent antimalarial activity [54]. Further, a deeper understanding of the role(s) of autophagy in the liver-stage parasites, especially the dormant stages (hypnozoites), is essential to enable discovery of novel anti-relapse drugs for the radical treatment of malaria. Additionally, the selective chemotherapeutic augmentation of host autophagy may also enhance protection against malaria. Nevertheless, it must be emphasized here that such approaches warrant a fine balance of caution and optimism.

\section{Acknowledgments}

We are grateful to Prof. K. K. Bhutani, Officiating Director, NIPER (National Institute of Pharmaceutical Education and Research), for his help and encouragement. P. C. is grateful to NIPER for financial assistance. This is NIPER communication No. 498.

\section{References}

[1] Fact Sheet World Malaria Report. 2015. Accessed January 4 , 2016. http://www.who.int/mediacentre/factsheets/fs094/en/.

[2] Calderaro, A., Piccolo, G., Gorrini, C., Rossi, S., Montecchini, S., Dell'Anna, M. L., De Conto, F., Medici, M. C., Chezzi, C., and Arcangeletti, M. C. 2013. "Accurate Identification of the Six Human Plasmodium spp. Causing Imported Malaria, Including Plasmodium ovale wallikeri and Plasmodium knowlesi." Malaria Journal 12 (1): 1-6.

[3] Lalremruata, A., Magris, M., Vivas-Martinez, S., Koehler, M., Esen, M., Kempaiah, P., Jeyaraj, S., Perkins, D. J., Mordmüller, B., and Metzger, W. G. 2015. "Natural Infection of Plasmodium brasilianum in Humans: Man and Monkey Share Quartan Malaria Parasites in the Venezuelan Amazon.” EBioMedicine 2 (9): 1186-92.

[4] Hyde, J. E. 2007. "Drug-Resistant Malaria-An Insight." FEBS Journal 274 (18): 4688-4698.

[5] Overview of Malaria Treatment. 2015. Accessed January 4 ,

2016. http://www.who.int/malaria/areas/treatment/overview/en/.

[6] Wongsrichanalai, C., and Meshnick, S. R. 2008. "Declining Artesunate-Mefloquine Efficacy against Falciparum Malaria on the Cambodia-Thailand Border." Emerging Infectious Diseases 14 (5): 716-9.

[7] Q\&A on Artemisinin Resistance. 2015. Accessed January 4, 2016. http://who.int/malaria/media/artemisinin_resistance_qa/en/.

[8] Levine, B., and Kroemer, G. 2008. "Autophagy in the Pathogenesis of Disease.” Cell 132 (1): 27-42.

[9] Hughes, T., and Rusten, T. E. 2008. Eukaryotic Membranes and Cytoskeleton: Origins and Evolution. Austin: Landes Bioscience, 111-8.

[10] Levine, B., and Yuan, J. 2005. "Autophagy in Cell Death: An Innocent Convict?" The Journal of Clinical Investigation 115 (10): 2679-88.

[11] Klionsky, D. J. 2008. "Autophagy Revisited: A Conversation with Christian de Duve." Autophagy 4 (6): 740-3.

[12] Mizushima, N. 2007. "Autophagy: Process and Function." Genes \& Development 21 (22): 2861-73.

[13] Takeshige, K., Baba, M., Tsuboi, S., Noda, T., and Ohsumi, Y. 1992. "Autophagy in Yeast Demonstrated with Proteinase-Deficient Mutants and Conditions for Its Induction.” The Journal of Cell Biology 119 (2): 301-11.

[14] Baehrecke, E. H. 2005. "Autophagy: Dual Roles in Life and Death?" Nature Reviews Molecular Cell Biology 6 (6): 505-10.

[15] Yang, Z., and Klionsky, D. J. 2010. "Eaten Alive: A History of Macroautophagy." Nature Cell Biology 12 (9): 814-22.

[16] Mintern, J. D., and Harris, J. 2015. "Autophagy and Immunity." Immunology and Cell Biology 93 (1): 1-2.

[17] De Brito, T., Barone, A., and Faria, R. 1969. "Human Liver Biopsy in P. falciparum and P. vivax Malaria." Virchows Archiv A 348 (3): 220-9.

[18] Abraham, R., and Hendy, R. 1970. "Effects of Chronic Chloroquine Treatment on Lysosomes of Rat Liver Cells." Experimental and Molecular Pathology 12 (2): 148-59. 
[19] Totino, P. R. R., Daniel-Ribeiro, C. T., Corte-Real, S., and Ferreira-da-Cruz, M. 2007. "Plasmodium falciparum: Erythrocytic Stages Die by Autophagic-Like Cell Death Under Drug Pressure." Experimental Parasitology 118 (4): 478-86.

[20] Klionsky, D. J. 2011. "The Autophagosome Is Overrated!" Autophagy 7 (4): 353-4.

[21] Tomlins, A. M., Ben-Rached, F., Williams, R. A., Proto, W. R., Coppens, I., Ruch, U., Gilberger, T. W., Coombs, G. H., Mottram, J. C., Müller, S., and Langsley, G. 2013. "Plasmodium falciparum ATG8 Implicated in Both Autophagy and Apicoplast Formation." Autophagy 9 (10): 1540-52.

[22] Eickel, N., Kaiser, G., Prado, M., Burda, P-C., Roelli, M., Stanway, R. R., and Heussler, V. T. 2013. "Features of Autophagic Cell Death in Plasmodium Liver-Stage Parasites." Autophagy 9 (4): 568-80.

[23] Hain, A. U., Bartee, D., Sanders, N. G., Miller, A. S., Sullivan, D. J., Levitskaya, J., Meyers, C. F., and Bosch, J. 2014. "Identification of an Atg8-Atg3 Protein-Protein Interaction Inhibitor from the Medicines for Malaria Venture Malaria Box Active in Blood and Liver Stage Plasmodium falciparum Parasites." Journal of Medicinal Chemistry 57 (11): 4521-31.

[24] Cervantes, S., Bunnik, E. M., Saraf, A., Conner, C. M., Escalante, A., Sardiu, M. E., Ponts, N., Prudhomme, J., Florens, L., and Le Roch, K. G. 2014. "The Multifunctional Autophagy Pathway in the Human Malaria Parasite, Plasmodium falciparum." Autophagy 10 (1): 80-92.

[25] Hain, A. U., and Bosch, J. 2013. "Autophagy in Plasmodium, a Multifunctional Pathway?" Computational and Structural Biotechnology Journal 8 (11): 1-9.

[26] Kitamura, K., Kishi-Itakura, C., Tsuboi, T., Sato, S., Kita, K., Ohta, N., and Mizushima, N. 2012. "Autophagy-Related Atg8 Localizes to the Apicoplast of the Human Malaria Parasite Plasmodium falciparum." PloS One 7 (8): e42977.

[27] Sinai, A. P., and Roepe, P. D. 2012. "Autophagy in Apicomplexa: A Life Sustainng Death Mechanism?" Trends in Parasitology 28 (9): 358-64.

[28] Gaviria, D., Paguio, M. F., Turnbull, L. B., Tan, A., Siriwardana, A., Ghosh, D., Ferdig, M. T., Sinai, A. P., and Roepe, P. D. 2013. “A Process Similar to Autophagy is Associated with Cytocidal Chloroquine Resistance in Plasmodium falciparum.” PloS One 8 (11): e79059.

[29] Chen, K., Shou, L. M., Lin, F., Duan, W. M., Wu, M. Y., Xie, X., Xie, Y. F., Li, W., and Tao, M. 2014. “Artesunate Induces G2/M Cell Cycle Arrest Through Autophagy Induction in Breast Cancer Cells." Anti-cancer Drugs 25 (62): 652-62.

[30] Wang, Z., Hu, W., Zhang, J. L., Wu, X. H., and Zhou, H. J.
2012. "Dihydroartemisinin Induces Autophagy and Inhibits the Growth of Iron-Loaded Human Myeloid Leukemia K562 Cells via ROS Toxicity." FEBS Open Bio 2 (1): 103-12.

[31] Golenser, J., Waknine, J. H., Krugliak, M., Hunt, N. H., and Grau, G. E. 2006. "Current Perspectives on the Mechanism of Action of Artemisinins." International Journal for Parasitology 36 (14): 1427-41.

[32] Mizushima, N., and Sahani, M. H. 2014. "ATG8 Localization in Apicomplexan Parasites: Apicoplast and More?" Autophagy 10 (9): 1487-94.

[33] Veletzky, L., Rehman, K., Lingscheid, T., Poeppl, W., Loetsch, F., Burgmann, H., and Ramharter, M. 2014. "In vitro Activity of Immunosuppressive Drugs Against $P$. falciparum." Malaria Journal 13 (1): 476.

[34] Walker, D. M. 2013. "The Study of Autophagy in Plasmodium falciparum." Ph.D. thesis, Ohio State University.

[35] Jayabalasingham, B., Bano, N., and Coppens, I. 2010. "Metamorphosis of the Malaria Parasite in the Liver is Associated with Organelle Clearance." Cell Research 20 (9): 1043-59.

[36] Seglen, P. O., and Gordon, P. B. 2013. "3-Methyladenine: Specific Inhibitor of Autophagic/Lysosomal Protein Degradation in Isolated Rat Hepatocytes." Proceedings of the National Academy of Sciences 79 (6): 1889-92.

[37] Münz, C. 2012. "Antigen Processing for MHC Class II Presentation via Autophagy." Frontiers in Immunology 3 (9): 1-6.

[38] Münz, C. 2015. "Of LAP, CUPS, and DRibbles-Unconventional Use of Autophagy Proteins for MHC Restricted Antigen Presentation." Frontiers in Immunology 6 (200): 1-5.

[39] Lee, H. K., Mattei, L. M., Steinberg, B. E., Alberts, P., Lee, Y. H., Chervonsky, A., Mizushima, N., Grinstein, S., and Iwasaki, A. 2010. "In vivo Requirement for Atg5 in Antigen Presentation by Dendritic Cells." Immunity 32 (2): 227-39.

[40] Deretic, V., Saitoh, T., and Akira, S. 2013. "Autophagy in Infection, Inflammation and Immunity." Nature Reviews Immunology 13 (10): 722-37.

[41] Ogawa, M., Yoshimori, T., Suzuki, T., Sagara, H., Mizushima, N., and Sasakawa, C. 2005. "Escape of Intracellular Shigella from Autophagy." Science 307 (5710): 727-31.

[42] Besteiro, S., Williams, R. A., Morrison, L. S., Coombs, G. H., and Mottram, J. C. 2006. "Endosome Sorting and Autophagy are Essential for Differentiation and Virulence of Leishmania major." Journal of Biological Chemistry 281 (16): 11384-96.

[43] Orvedahl, A., Alexander, D., Tallo'czy, Z., Sun Q, Wei Y, Zhang, W., Burns, D., Leib, D. A., and Levine, B. 2007. 
"HSV-1 ICP34.5 Confers Neurovirulence by Targeting the Beclin 1 Autophagy Protein." Cell Host \& Microbe 1 (1): 23-35.

[44] Richie, D. L., Fuller, K. K., Fortwendel, J., Miley, M. D., McCarthy, J. W., Feldmesser, M., Rhodes, J. C., and Askew, D. S. 2007. "Unexpected Link Between Metal Ion Deficiency and Autophagy in Aspergillus fumigatus." Eukaryotic Cell 6 (12): 2437-47.

[45] Deretic, V. 2014. "Autophagy in Tuberculosis." Cold Spring Harbor Perspectives in Medicine 4 (11): a018481.

[46] Gomes, L. C., and Dikic, I. 2014. "Autophagy in Antimicrobial Immunity." Molecular Cell 54 (2): 224-33.

[47] Delgado, M. A., Elmaoued, R. A., Davis, A. S., Kyei, G., and Deretic ,V. 2008. "Toll-like Receptors Control Autophagy." The EMBO Journal 27 (7): 1110-21.

[48] Levine, B., Mizushima, N., and Virgin, H. W. 2011. "Autophagy in Immunity and Inflammation." Nature 469 (7330): 323-35.

[49] Orvedahl, A., and Levine, B. 2009. "Eating the Enemy Within: Autophagy in Infectious Diseases." Cell Death \& Differentiation 16 (1): 57-69.

[50] Prado, M., Eickel, N., De Niz, M., Heitmann, A., Nersesian, C., Wacker, R., Schmuckli-Maurer, J., Caldelari, R., Janse, C. J., Khan, S. M., and May, J. 2015. "Long-term Live Imaging Reveals Cytosolic Immune
Responses of Host Hepatocytes Against Plasmodium Infection and Parasite Escape Mechanisms." Autophagy 11 (9): 1561-79.

[51] Van Dijk, M. R., Douradinha, B., Franke-Fayard, B., Heussler, V., van Dooren, M. W., van Schaijk, B., van Gemert, G. J., Sauerwein, R. W., Mota, M. M., Waters, A. P., and Janse, C. J. 2005. "Genetically Attenuated, P36p-Deficient Malarial Sporozoites Induce Protective Immunity and Apoptosis of Infected Liver Cells." Proceedings of the National Academy of Sciences of the United States of America 102 (34): 12194-9.

[52] Mejia, P., Villarreal, J., Hine, C., Harputlugil, E., Lang, S., Calay, E., Rogers, R., Wirth, D., Duraisingh, M. T., and Mitchell, J. R. 2015. "Dietary Restriction Protects Against Experimental Cerebral Malaria via Leptin Modulation and T-cell mTORC1 Suppression." Nature Communications 6 (6050): 1-13.

[53] Kaushansky, A., Albert, S. Y., Austin, L. S., Mikolajczak, S. A., Vaughan, A. M., Camargo, N., Metzger, P. G., Douglass, A. N., MacBeath, G., and Kappe, S. H. 2013. "Suppression of Host p53 is Critical for Plasmodium Liver-stage Infection." Cell Reports 3 (3): 630-7.

[54] Tesfaye, S., Prakash, B., and Singh, P. P. 2015. "Apicoplast Biosynthetic Pathways as Possible Targets for Combination Therapy of Malaria." Journal of Pharmacy and Pharmacology 3 (1): 101-15. 